\title{
A note on the existence of invariant punctured tori in the planar circular restricted three-body problem
}

\author{
A. CHENCINER AND J. LLIBRE \\ Département de Mathématiques, Université Paris VII, F-75251 Paris, France; \\ Departament de Matemàtiques, Facultat de Cièncias, Universidad Autònoma de \\ Barcelona, E-08193 Barcelona, Spain
}

Abstract. The existence of transversal ejection-collision orbits in the restricted three-body problem is shown to imply, via the KAM theorem, the existence, for certain intervals of (large) values of the Jacobi constant, of an uncountable number of invariant punctured tori in the corresponding (non-compact) energy surface. The proof is based on a comparison between Levi-Civita and McGehee regularizing variables. That these transversal ejection-collision orbits do actually exist was proved in [5] in the case where one of the primaries has a small mass and the zero-mass body revolves around the other (and for all values of the Jacobi constant compatible with the existence of three connected components for the Hill region); it is proved here without any restriction on the masses, well in the spirit of Conley's thesis [3].

Part of our setting and notations are collected in figure 1; they are essentially those of [2], [3] and [5].

The position of the zero-mass body in the moving frame is given by the complex number $x$; in the variables $x, y=d x / d t+i \omega x$, (one half of) the Jacobi constant becomes

$$
H(x, y)=|y|^{2}+i(\bar{x} y-x \bar{y})-2 \nu /|x|-2 \mu /|x+1|-\mu(x+\bar{x})+2 \mu,
$$

and Newton's equations read

$$
d x / d t=\partial H / \partial \bar{y}, \quad d y / d t=-\partial H / \partial \bar{x} .
$$

(We have used the usual normalization $G=1, \mu+\nu=1$, which implies $\omega=1$, and chosen the same constant $2 \mu$ as Conley.)

The value $-1 / \varepsilon^{2}$ of $H$ (which is taken large and negative) defines our main parameter $\varepsilon$. If $\varepsilon$ is small enough, there are three Hill regions and we shall suppose (as Conley does) that the zero-mass body rotates around the primary of mass $\nu$. Notice that the limit situation $\varepsilon=0$ corresponds to a collision. $\dagger$ Fixing $\varepsilon$, we regularize this collision (as Conley did) using Levi-Civita variables

$$
x=2 z^{2}, \quad y=w / \varepsilon \bar{z}, \quad d t=2 \varepsilon|x| d t^{\prime}
$$

† For a general view of collision problems see [6]. 


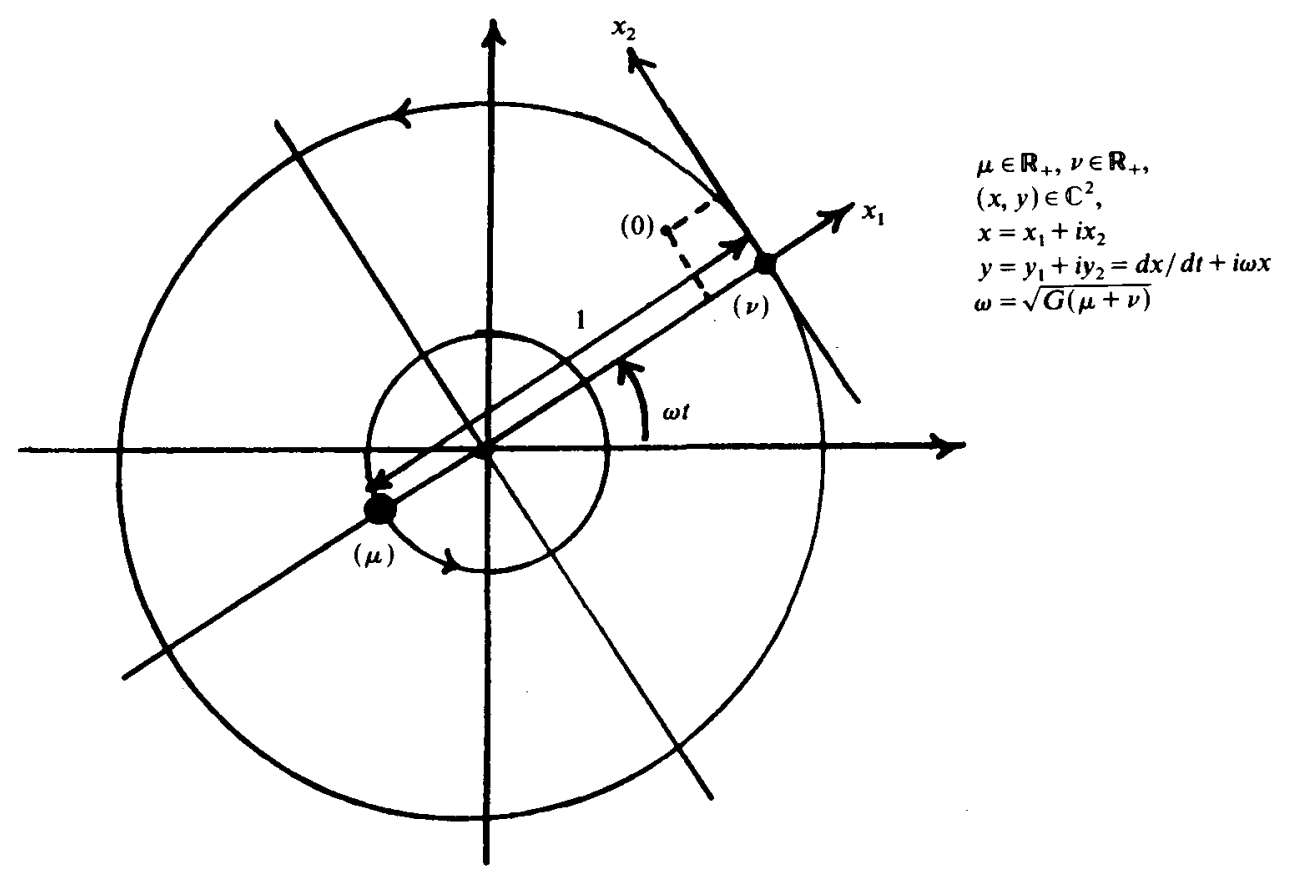

Figure 1

and get the new equations (in $\mathbb{R}^{4}=\mathbb{C}^{2}$ )

$$
\begin{gathered}
d z / d t^{\prime}=\partial K / \partial \bar{w}, \quad d w / d t^{\prime}=-\partial K / \partial \bar{z}, \\
K(z, w)=\{1+2 i \varepsilon(\bar{z} w-z \bar{w})\}|z|^{2}+|w|^{2}-\nu \varepsilon^{2}-\mu \varepsilon^{3} g(z), \\
g(z)=2|z|^{2}\left\{1 /\left|2 z^{2}+1\right|-1+z^{2}+(\bar{z})^{2}\right\}=|z|^{2}\left\{2|z|^{4}+3\left(z^{4}+(\bar{z})^{4}\right)+O\left(|z|^{6}\right)\right\} .
\end{gathered}
$$

These new equations have the following property: the energy surface $K=0$ is close to (and diffeomorphic to) the 3-sphere $|z|^{2}+|w|^{2}=\nu \varepsilon^{2}$; if we restrict the Levi-Civita mapping $(z, w) \rightarrow(x, y)$ to the complement (diffeomorphic to an open solid torus) of the 'circle' $z=0$, we get an orbit preserving twofold covering of the energy surface $H=-1 / \varepsilon^{2}$ of the restricted three-body problem.

The structure of $K$ is nice enough. If one keeps only the leading (quadratic) terms, the linear flow one obtains (Hopf flow) is, up to the twofold covering, the usual regularization of the two-body problem by the geodesic flow on the round 2-sphere ([2], [7]). If one forgets only the last term $\mu \varepsilon^{2} g(z)$ (which is of order 6 in $z$ ), one obtains the still integrable two-body problem in a rotating frame: the complement in the energy surface of two linked periodic orbits (corresponding to the direct and retrograde circular motions having the given value of the Jacobi integral) is foliated by invariant tori parametrized by the angular momentum. The 'middle' torus, corresponding to zero angular momentum, contains the 'circle' $z=0$ and each integral curve lying on this torus is made up of ejection-collision orbits (figure 2). 


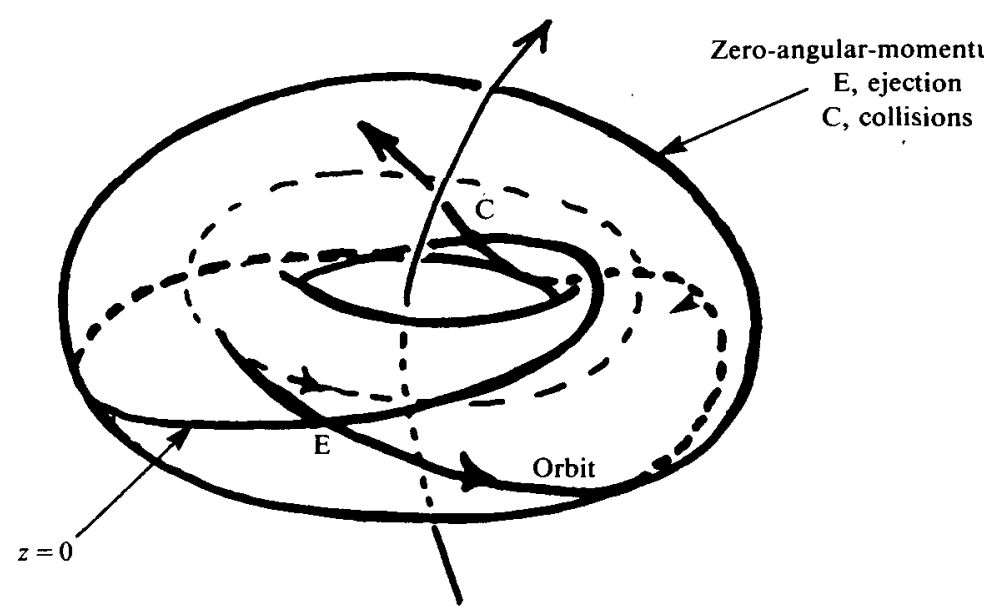

FIGURE 2

When the perturbation $\mu \epsilon^{2} g(z)$ is added, the two periodic orbits associated to circular movements continue to exist [3]: they now correspond to quasi-circular direct and retrograde motions of the zero-mass body in the rotating frame. As in [3], one can take these orbits as the boundary of an annulus of section; moreover, one can choose this annulus so as to contain the 'circle' $z=0$ (figure 3; also compare with [1]). Our results will be stated in terms of the Poincaré first return map $P_{f}$ on such an annulus $A$.

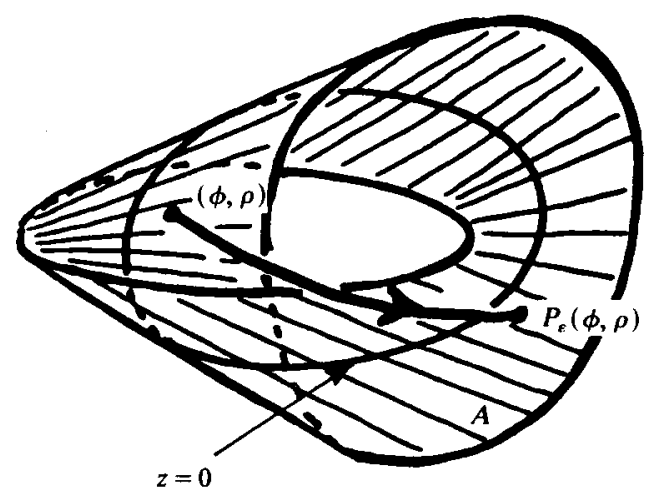

Figure 3

THEOREM 1. If $\mu \nu \neq 0$ and $\varepsilon$ is small enough; the intersection of the 'circle' $z=0$ with its image under $P_{\varepsilon}$ consists of exactly eight transversal points.

THEOREM 2. If $\mu \nu \neq 0$, in any neighbourhood of $\varepsilon=0$ in $\mathbb{R}^{+}$, there exists an interval of values of $\varepsilon$ such that the 'circle' $z=0$ intersects an uncountable number of invariant curves of $P_{\varepsilon}$, each in a finite number of points. Moreover, in each of these intervals there are at least two values of $\varepsilon$ such that the 'circle' $z=0$ contains a pair of points $\alpha, P_{\varepsilon}(\alpha)$ belonging to the same invariant curve of $P_{F}$. 
The interpretation of theorem 2 is clearly that, for a certain set of large values of Jacobi constant, the original problem admits invariant punctured tori which can even contain ejection-collision orbits (see figure 8).

Proof of theorem 1. We blow up $z=0$ by choosing polar coordinates in a transversal section:

$$
z=\varepsilon r e^{i \theta}, \quad \chi \equiv v+i u=r w / z, \quad d t^{\prime}=r d t^{\prime \prime} .
$$

This amounts to adding a 2-torus boundary to the complementary open torus and leads naturally to McGehee-like coordinates (compare with [5]); the transformed equations read

$$
\begin{aligned}
& d r / d t^{\prime \prime}=r v \\
& d \theta / d t^{\prime \prime}=u-2 \varepsilon^{3} r^{3}, \\
& d u / d t^{\prime \prime}=-u v+\mu \operatorname{Im}\{\bar{z}(\partial g / \partial \bar{z})\}, \\
& d v / d t^{\prime \prime}=u^{2}-r^{2}+4 u \varepsilon^{3} r^{3}+\mu \operatorname{Re}\{\bar{z}(\partial g / \partial \bar{z})\},
\end{aligned}
$$

with the integral

$$
\left(1 / \varepsilon^{2}\right) K=u^{2}+v^{2}-\nu+r^{2}-4 \varepsilon^{3} r^{3} u-\mu g(z),
$$

so that the compactified energy surface corresponding to $H=-1 / \varepsilon^{2}$ becomes the solid torus of equation

$$
u^{2}+v^{2} \leq \nu \text {. }
$$

As we could guess, the flow on and near the boundary (the collision manifold) is qualitatively the same as in the McGehee regularization of the Kepler problem ([4], [5]); it is depicted in figure 4, which shows the two circles of equilibria $C_{+}$ and $C_{-}$on the torus $u^{2}+v^{2}=\nu$ and their asymptotic trajectories $W_{+}$and $W_{-}$.

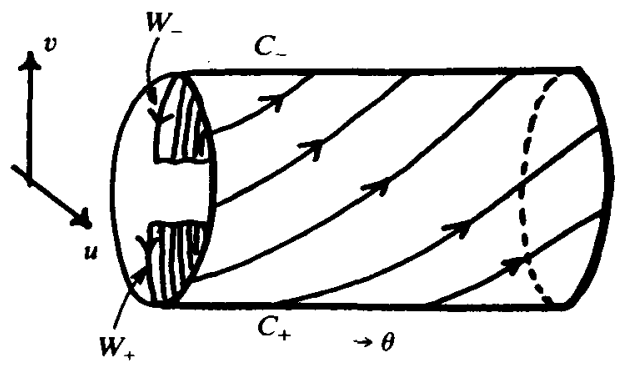

Figure 4

The assertion of theorem 1 is obviously equivalent to the existence of eight 'simple' transversal ejection-collision orbits, i.e. orbits going 'directly' (without following part of the boundary torus) from one circle of equilibria to the other, along which $W_{+}$and $W_{-}$intersect transversally. Without the perturbation coming from the terms $\mu \varepsilon^{2} g(z)$, we get an integrable situation in which trajectories of this kind generate an annulus $W_{+}=W_{-}$bordered by the circles of equilibria; in the Levi-Civita variables, the union of this annulus with the 'circle' $z=0$ is nothing but the 
zero-angular-momentum invariant torus, and the corresponding first return map $\boldsymbol{P}_{\boldsymbol{\varepsilon}}$ leaves $z=0$ invariant. The proof of theorem 1 will be obtained by perturbation from the even simpler integrable case $\varepsilon=0$, for which we easily compute explicitly the solutions belonging to $W_{+}=W_{-}$. Indeed, because of the integral $u^{2}+v^{2}-\nu+r^{2}=0$, the restriction of the system to $W_{+}=W_{-}$, whose equation is then $u=0$, can be written

$$
d r / d t^{\prime \prime}=r v, \quad d \theta / d t^{\prime \prime}=0, \quad d v / d t^{\prime \prime}=v^{2}-\nu,
$$

and the solution with initial conditions

$$
u_{0}(0)=0, \quad v_{0}(0)=0, \quad r_{0}(0)=\sqrt{\nu}, \quad \theta_{0}(0)=\theta_{0}
$$

is seen to be

$$
\begin{gathered}
r_{0}\left(t^{\prime \prime}\right)=(\sqrt{\nu}) / \cosh \left(\sqrt{\nu} t^{\prime \prime}\right), \quad \theta_{0}\left(t^{\prime \prime}\right)=\theta_{0}, \quad u_{0}\left(t^{\prime \prime}\right)=0, \\
v_{0}\left(t^{\prime \prime}\right)=-\sqrt{\nu} \tanh \left(\sqrt{\nu} t^{\prime \prime}\right) .
\end{gathered}
$$

When $\varepsilon>0$, we call $r_{\varepsilon}, \theta_{\varepsilon}, u_{\varepsilon}, v_{\varepsilon}$, the unique solution in $W_{+}$defined by $\theta_{\varepsilon}(0)=\theta_{0}$, $v_{\varepsilon}(0)=0$. Such a solution is well defined and unique because, for $\varepsilon$ small enough, the part of $W_{+}$in the region $v \leq 0$ intersects transversally the annulus $v=0$ along a curve $u=\phi(\theta),\|\phi\|_{0}=O(\varepsilon)$. By an argument similar to that of [8], one sees that this solution approximates uniformly the former one on the whole interval of time $[0,+\infty[:$

$$
\begin{aligned}
& r_{\varepsilon}\left(t^{\prime \prime}\right)=r_{0}\left(t^{\prime \prime}\right)+\varepsilon^{a} r_{1}\left(t^{\prime \prime}\right)+O\left(\varepsilon^{a+1}\right), \\
& \theta_{\varepsilon}\left(t^{\prime \prime}\right)=\theta_{0}\left(t^{\prime \prime}\right)+\varepsilon^{b} \theta_{1}\left(t^{\prime \prime}\right)+O\left(\varepsilon^{b+1}\right), \\
& u_{\varepsilon}\left(t^{\prime \prime}\right)=u_{0}\left(t^{\prime \prime}\right)+\varepsilon^{c} u_{1}\left(t^{\prime \prime}\right)+O\left(\varepsilon^{c+1}\right), \\
& v_{\varepsilon}\left(t^{\prime \prime}\right)=v_{0}\left(t^{\prime \prime}\right)+\varepsilon^{d} v_{1}\left(t^{\prime \prime}\right)+O\left(\varepsilon^{d+1}\right),
\end{aligned}
$$

where the $O$ are uniform, $\theta_{1}(0)=0, v_{1}(0)=0$ and, when $t^{\prime \prime} \rightarrow+\infty$,

$$
\lim r_{1}\left(t^{\prime \prime}\right)=\lim u_{1}\left(t^{\prime \prime}\right)=\lim v_{1}\left(t^{\prime \prime}\right)=0 \text {. }
$$

In the sequel it will be simpler to work with the new time $s=\sqrt{\nu} t^{\prime \prime}$; the derivative with respect to $s$ will be denoted by and the same notation $r_{1}, \ldots$, will be used when these functions are considered as functions of $s$.

One first notices that $c$ must be at least 6 in order to have $u_{1} \neq 0$, otherwise $d u_{1} / d t^{\prime \prime}=-v_{0} u_{1}$, i.e. $\dot{u}_{1}=(\tanh s) u_{1}$ or $u_{1}=\gamma \cosh s$, where the constant $\gamma$ must be zero if $u_{1}$ is to have limit 0 at $s \rightarrow+\infty$. Now, with $c=6$, one gets

$$
d u_{1} / d t^{\prime \prime}=-v_{0} u_{1}+\left[\left(\mu / \varepsilon^{6}\right) \operatorname{Im}\{\bar{z}(\partial g / \partial \bar{z})\}\right]_{\varepsilon=0},
$$

i.e.

$$
\dot{u}_{1}=(\tanh s) u_{1}-12(\mu / \sqrt{\nu})\left(r_{0}\right)^{6} \sin 4 \theta_{0} .
$$

Finally, as $u_{1} \rightarrow 0$ when $s \rightarrow+\infty$,

$$
u_{1}=12 \mu(\nu)^{5 / 2}\left(\int_{s}^{+\infty}\left(1 / \cosh ^{7} x\right) d x\right) \sin 4 \theta_{0} \cosh s .
$$

From this we conclude that the intersection with the annulus $v=0$ of the part of $W_{+}$below it is the graph of a function $\theta \mapsto u=\phi(\theta)$, where

$$
\phi(\theta)=12 \mu(\nu)^{5 / 2}\left(\int_{0}^{+\infty}\left(1 / \cosh ^{7} x\right) d x\right) \varepsilon^{6} \sin 4 \theta+O\left(\varepsilon^{7}\right) .
$$


Using the invariance of our equations under the transformation

$$
\left(\theta, r, u, v, t^{\prime \prime}\right) \mapsto\left(-\theta, r, u,-v,-t^{\prime \prime}\right),
$$

we get that the intersection of the same annulus $v=0$ with the part of $W_{-}$above it is nothing but the graph of $\theta \mapsto \phi(-\theta)$.

As the intersection of these two curves is obviously composed (for $\varepsilon$ small enough) of eight transversal points, the conclusion follows (figure 5).

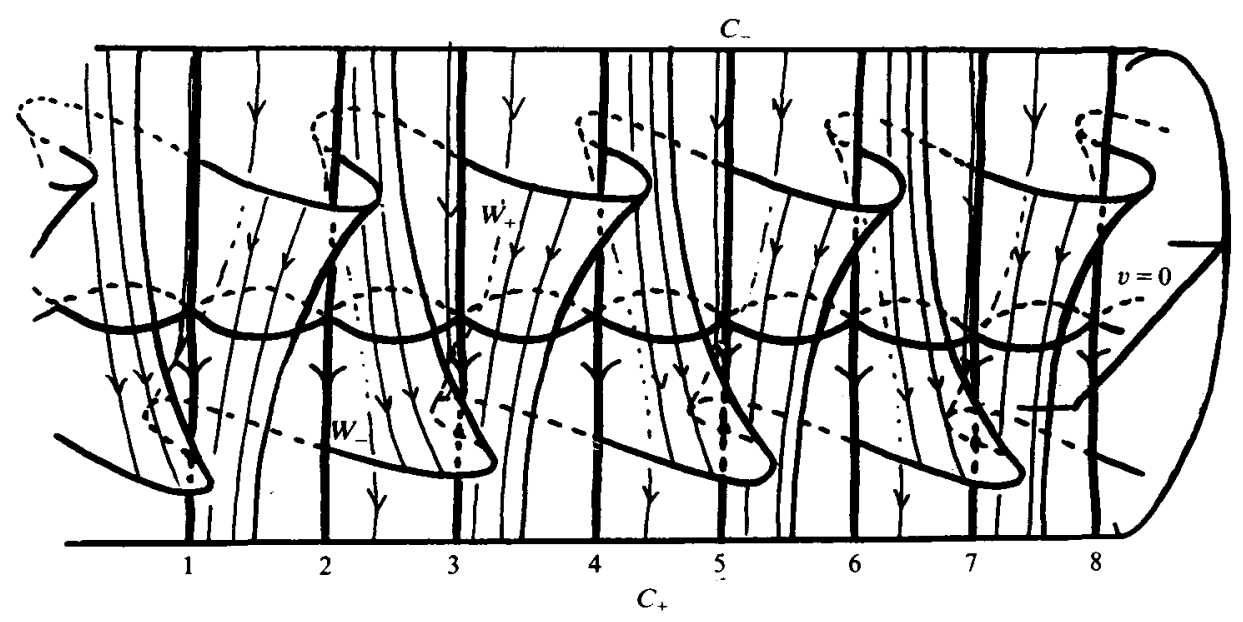

FIGURE 5. $\mu>0$.

Remarks. (i) Because of the twofold covering involved in the Levi-Civita variables, these eight points correspond to only four points in the McGehee compactification of the original problem which appears in [5] (invariance of the equations under $\theta \rightarrow \theta+\pi)$.

(ii) The annulus $v=0$, which plays such a prominent role in this proof, is closely related to the annulus $A$. Indeed, if we set (see [2] and [3])

$$
\xi_{1}=w+i f z, \quad \xi_{2}=\bar{w}+i f \bar{z}, \quad f^{2}=1+2 i \varepsilon(\bar{z} w-z \bar{w}),
$$

we get

$$
v=\left[1 /\left(\varepsilon\left|\xi_{1}-\bar{\xi}_{2}\right|\right)\right] \operatorname{Im}\left(\xi_{1} \xi_{2}\right), \quad u=\left[1 /\left(2 \varepsilon\left|\xi_{1}-\bar{\xi}_{2}\right|\right)\right]\left(\left|\xi_{1}\right|^{2}-\left|\xi_{2}\right|^{2}\right)
$$

In particular, $v=0$ is equivalent to $\operatorname{Arg} \xi_{1}+\operatorname{Arg} \xi_{2}=0(\bmod \pi)$; but the same equation $\bmod 2 \pi$, which corresponds to $v=0,|u| \geq \sqrt{\nu / 2}$, is just the annulus $A$ when $\mu=0$ and is very close to the actual annulus $A$ in the general case (see [2] and [3]).

Finally, $u=0$ is equivalent to $\left|\xi_{1}\right|^{2}-\left|\xi_{2}\right|^{2}=0$, which is of course the zero-momentum invariant torus in the case $\mu=0$. So, in this case, $W_{+}=W_{-}$has the equation $u=0$, happily compatible with our calculations.

We have depicted in figure 6 the (integrable) flow when $\mu=0$.

(iii) Finiteness in theorem 1 already follows from analyticity; indeed, it is not difficult to prove that $P_{\varepsilon}$ is real analytic up to the boundary for the differential structure on the annulus which comes from its embedding in the energy sphere. On 


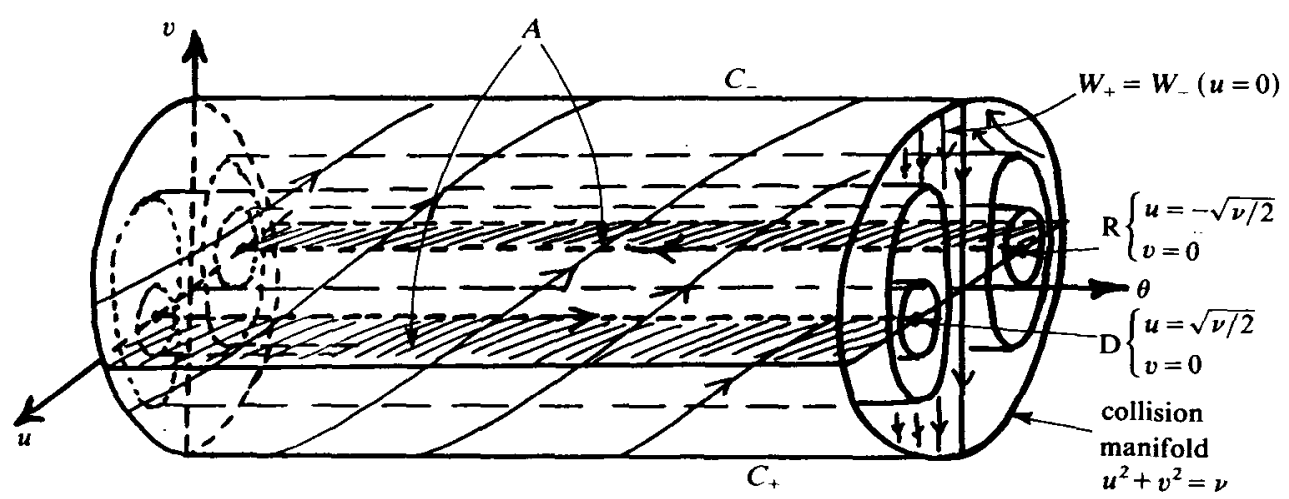

FIGURE 6. $\mu=0 . A$ is bounded by the direct (D) and retrograde (R) circular periodic orbits.

each component of the boundary it is even analytically conjugate to a rotation whose angle can be simply deduced from the derivative at its fixed point of the return map defined by the corresponding periodic orbit on a transversal section.

We now come to the proof of theorem 2. In a course [2] based on Conley's thesis, the first author computed a truncated normal form of the Poincare return map on the annulus $A$ in coordinates $\phi, \rho$ which identifies $A$ to a subannulus of $(\mathbb{R} / \mathbb{Z}) \times \mathbb{R}$ whose boundaries are approximately given by $|\rho|=1$ :

$$
P_{\varepsilon}(\phi, \rho)=\left\{\phi+\frac{1}{2}-\nu \varepsilon^{3} / 2-\frac{3}{2}(1-\mu / 4) \nu^{2} \varepsilon^{6} \rho+O\left(\varepsilon^{7}\right), \rho+O\left(\varepsilon^{7}\right)\right\}
$$

in these coordinates the 'circle' $z=0$ appears as the graph of $\zeta: \mathbb{R} / \mathbb{Z} \rightarrow \mathbb{R}$ whose $C^{0}$ norm $\|\zeta\|_{0}$ is $O\left(\varepsilon^{3}\right)$.

Remark 1. Though very natural, the coordinates $\phi, \rho$ are analytic in the interior but not differentiable up to the boundary; this will cause no trouble in the sequel.

Remark 2. Theoretically, a normal form of $P_{\varepsilon}$ could be pushed further on, but we did explicit calculations only to this order, which is enough for what follows.

Let us fix $\varepsilon_{0}>0$ and $\beta \geq 0$; there certainly exists (an uncountable number of) $\varepsilon_{1}$ such that

$$
\begin{gathered}
0<\varepsilon_{1}<\varepsilon_{0} / 2, \\
\forall p / q, \quad\left|(\nu / 2)\left(\varepsilon_{1}\right)^{3}-\frac{1}{2}-p / q\right| \geq\left[\frac{3}{2}(1-\mu / 4) \nu^{2}\left(\varepsilon_{1}\right)^{6}\right] / q^{2+\beta} .
\end{gathered}
$$

If $\varepsilon$ belongs to the interval $\left[\varepsilon_{1}^{\prime}, \varepsilon_{1}^{\prime \prime}\right]$ defined by

$$
-(\nu / 2) \varepsilon^{3}-(1-\mu / 4) \nu^{2} \varepsilon^{6} \leq-(\nu / 2)\left(\varepsilon_{1}\right)^{3} \leq-(\nu / 2) \varepsilon^{3}+(1-\mu / 4) \nu^{2} \varepsilon^{6},
$$

we set $\rho=r_{1}+\sigma$, where $r_{1}=r_{1}(\varepsilon)$ is defined by

$$
-(\nu / 2) \varepsilon^{3}-\frac{3}{2}(1-\mu / 4) \nu^{2} \varepsilon^{6} r_{1}=-(\nu / 2)\left(\varepsilon_{1}\right)^{3} \text {; }
$$

notice that $\left|r_{1}\right| \leq \frac{2}{3}$. In the new variables $(\phi, \sigma)$, the return map becomes

$$
P_{\varepsilon}(\phi, \sigma)=\left\{\phi+\frac{1}{2}-(\nu / 2)\left(\varepsilon_{1}\right)^{3}-\frac{3}{2}(1-\mu / 4) \nu^{2} \varepsilon^{6} \sigma+O\left(\varepsilon^{7}\right), \sigma+O\left(\varepsilon^{7}\right)\right\}
$$

and is certainly defined for $|\sigma| \leq \frac{1}{4}$.

We recall now the statement of the invariant curve theorem in a form essentially given in [9]. 
THEOREM. Let $0<\gamma \leq 1, C>0, \beta \geq 0$ be three real numbers, $\omega$ a real number satisfying $\forall p / q,|\omega-p / q| \geq \gamma C /|q|^{2+\beta}$, and $F$ a real analytic embedding of $(\mathbb{R} / \mathbb{Z}) \times\left[-\frac{1}{4}, \frac{1}{4}\right]$ into $(\mathbb{R} / \mathbb{Z}) \times \mathbb{R}$

$$
F(\phi, \sigma)=\left\{\phi+\omega+\gamma \sigma+\gamma \Phi_{1}(\phi, \sigma), \sigma+\gamma \Phi_{2}(\phi, \sigma)\right\}
$$

Suppose that $F$ has the 'intersection property' (i.e. any embedded closed curve going around the annulus must intersect its image under $F$ ) and consider a neighbourhood $\mathscr{A}=\left\{(\phi, \sigma),|\operatorname{Im} \phi| \leq a, \sigma \in \mathscr{A}^{\prime}\right\}$ of $(\mathbb{R} / \mathbb{Z}) \times\left[-\frac{1}{4}, \frac{1}{4}\right]$ in $(\mathbb{C} / \mathbb{Z}) \times \mathbb{C}$ on which the complex extension of $F$ is defined. For each $\eta>0$ there is a $\delta>0$ depending on $C, \beta, \mathscr{A}$ but not on $\gamma$, such that, if the $C^{0}$ norms on $\mathscr{A}$ of $\Phi_{1}$ and $\Phi_{2}$ satisfy $\left\|\Phi_{1}\right\|_{\mathscr{A}}+\left\|\Phi_{2}\right\|_{\mathscr{A}}<\delta$, there exists a unique real analytic function $\psi: \mathbb{R} / \mathbb{Z} \rightarrow\left[-\frac{1}{4}, \frac{1}{4}\right]$ whose graph is an invariant curve of $F$ on which $F$ is analytically conjugate to the rotation $\phi \rightarrow \phi+\omega$, and such that $\|\psi\|_{0}<\eta\left(\|\|_{0}\right.$ is the $C^{0}$ norm $)$.

In the case where $F=P_{\varepsilon}$, one has (after the replacement of $\phi b y-\phi$ )

$$
\begin{gathered}
\omega=-\frac{1}{2}+(\nu / 2)\left(\varepsilon_{1}\right)^{3}, \quad \gamma=\frac{3}{2}(1-\mu / 4) \nu^{2} \varepsilon^{6}, \\
\Phi_{i}(\phi, \sigma)=\varepsilon \Delta_{i}(\phi, \sigma, \varepsilon), \quad i=1,2,
\end{gathered}
$$

where $\Delta_{1}$ and $\Delta_{2}$ are bounded on a certain complex domain $\mathscr{A}$ independently of $\varepsilon$.

Now recall that $P_{\varepsilon}$ is a homeomorphism onto itself of a closed annulus $A$ containing $(\mathbb{R} / \mathbb{Z}) \times\left[-\frac{1}{2}, \frac{1}{2}\right]$ in its interior and that, because of its Hamiltonian origin, it preserves a measure which gives a non-zero weight to every open set: obviously this implies the 'intersection property'.

So the theorem applies with, say, $\eta=\frac{1}{6}$ as soon as $\varepsilon\left\|\Delta_{i}\right\|_{\mathscr{A}} \leq \delta$, which is obviously realized if $\varepsilon$ is small enough.

Finally, if we allow $\varepsilon$ to go through its allowed interval $\left[\varepsilon_{1}^{\prime}, \varepsilon_{1}^{\prime \prime}\right]$ of length $O\left[\left(\varepsilon_{1}\right)^{4}\right]$ centred on $\varepsilon_{1}$, we obtain a function $\Sigma_{\varepsilon}=r_{1}(\varepsilon)+\psi_{\varepsilon}$, depending continuously on $\varepsilon$ (this is a consequence of unicity in the theorem), whose graph (in coordinates $\phi$, $\rho$ ) is invariant under $P_{\varepsilon}$, and such that

$$
\begin{array}{ll}
\text { for } \varepsilon=\varepsilon_{1}^{\prime}, & \Sigma_{\varepsilon} \geq \frac{2}{3}-\frac{1}{6}=\frac{1}{2}, \\
\text { for } \varepsilon=\varepsilon_{1}^{\prime \prime}, & \Sigma_{\varepsilon} \leq-\frac{2}{3}+\frac{1}{6}=-\frac{1}{2} .
\end{array}
$$

The conclusion of theorem 1 now makes clear the existence of a set of values of $\varepsilon$, containing at least a subinterval of $\left[\varepsilon_{1}^{\prime}, \varepsilon_{1}^{\prime \prime}\right]$, for which the graph of $\Sigma_{\varepsilon}$ intersects the 'circle' $z=0$ (figure 7). As for theorem 1, the finiteness assertion comes from analyticity.

The last statement in theorem 2 comes from the following.

Lemma. Given (1) two closed curves 'going around' an annulus, depending continuously on a parameter and crossing each other as above, (2) a family of homeomorphisms of one of the curves onto itself depending on the same parameter, there exist at least two values of the parameter for which two intersection points of the curves are images of one another under the homeomorphism.

Proof. In the annulus of coordinates $\phi, \varepsilon$ ( $\phi$ parametrizes the curve on which the homeomorphism is defined), the set of intersection points (for all values of $\varepsilon$ ) has the homology of a circle $\varepsilon=$ constant. The conclusion comes from the (obvious) 

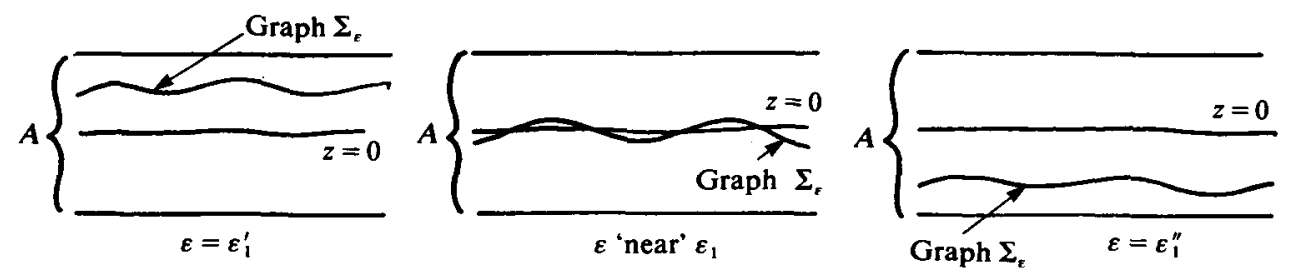

FIGURE 7

existence of at least two points of intersection of this set with its image under the homeomorphism $(\phi, \varepsilon) \rightarrow\left(f_{\varepsilon}(\phi), \varepsilon\right)$, where $f_{\varepsilon}$ is the given family of homeomorphisms.

Final comments. (1) As was pointed out to us by C. Simo, it is easy to see that if in the Hamiltonian $K$ we replace $g(z)$ by $|z|^{2}\left\{2|z|^{4}+3\left(z^{4}+(\bar{z})^{4}\right)\right\}$, and $\mu$ and $\nu$ by 1 , we get the so-called Hill's problem. Since only this part of the Taylor expansion of $g$ is used in our proofs, our results are also valid for Hill's problem.

(2) It is noticeable that the complicated dynamics we get in the rotating plane is still perfectly compatible with complete integrability and reflects only the relative position of the 'circle' $z=0$ with respect to eventual invariant tori in the Levi-Civita 3-sphere. Figure 8, drawn in the McGehee solid torus (up to a linear change of

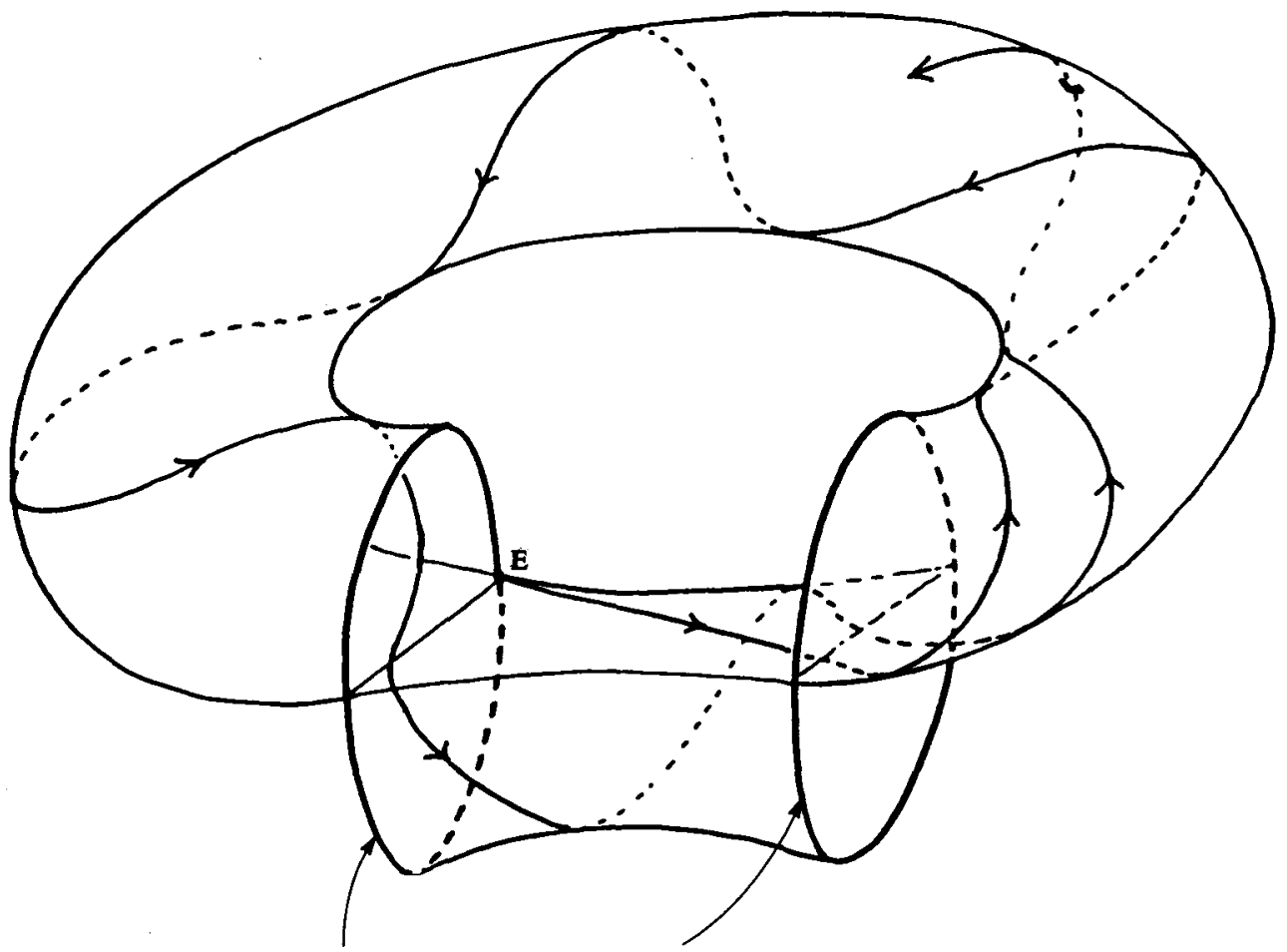

Two circles 'at infinity' (i.e. on the collision manifold); their complement is homeomorphic to $\left(T^{2}-2\right.$ points)

FIGURE 8. This figure corresponds to a case where $z=0$ would intersect transversally in two points an invariant torus of the system in the 3-sphere of Levi-Civita; we have represented an ejection trajectory. 
coordinates), is supposed to make this clear. On the other hand, it could happen that $z=0$ lies in a domain of instability. What features of the dynamics in the moving frame could detect this are still unclear.

Acknowledgements. This work was started during a visit of the second author to the Ecole Polytechnique and University of Paris VII and completed during a visit of the first author to the Centre de Recerca Matemàtica de Barcelona. The second author was partially supported by CAYCIT.

\section{REFERENCES}

[1] G. D. Birkhoff. Sur le problème restreint des trois corps (1er mémoire). Annali Scuola Normale Superiore de Pisa, S.2 4 (1935), 267-306.

[2] A. Chenciner. Le problème de la lune et la théorie des systèmes dynamiques, lère partie. Preprint. Université Paris VII.

[3] C. Conley. On some new long periodic solutions of the plane restricted three body problem. Commun. Pure Appl. Math. XVI (1963), 449-467.

[4] R. L. Devaney. Singularities in classical mechanical systems. In Ergodic Theory and Dynamical Systems I, Proceedings Special Year, Maryland 1979-80, ed. A. Katok. Birkhäuser, Basel (1981), 221-333.

[5] E. Lacomba \& J. Llibre. Transversal ejection-collision orbits for the restricted problem and the Hill's problem with applications. J. Differential Equations. To be published.

[6] R. McGehee. Singularities in classical celestial mechanics. Proc. ICM, Helsinki (1978), 827-834.

[7] J. K. Moser. Regularization of Kepler's problem and the averaging method on a manifold. Commun. Pure Appl. Math. XXIII (1970), 609-636.

[8] J. A. Sanders. Melnikov's method and averaging. Celestial Mech. 28 (1982), 171-181.

[9] C. L. Siegel \& J. K. Moser. Lectures on Celestial Mechanics. Springer, New York (1971). 